\title{
Walking Efficiency and Crossing Obstacle Ability of Variable Eccentric Wheel-legged Robot
}

\author{
Jie Huang a, Yujun Wang ${ }^{\text {b, * }}$, Can Fang ${ }^{c}$, Min Yi and Guiping Lan \\ School of Computer and Information Science, Southwest University, Chongqing 400715, China \\ ahuangjieperson@163.com, b774872400@qq.com, ccanfang@swu.edu.cn
}

\begin{abstract}
Keywords: mobile robot, variable eccentricity, double eccentric circle, walking efficiency, obstacle performance.
\end{abstract}

\begin{abstract}
Concerning that traditional mobile robot suffers the low walking efficiency or poor climbing ability, a new model of Variable Eccentric Wheel-legged Robot is proposed in this paper. The modeling and analysis of the kinematics and kinetics prove that the idea is feasible. The Variable Eccentric Wheel-legged Robot can not only effectively walk on a flat ground, but also own better climbing ability on a non-flat surface, comprising the obstacle of continuous steps. Firstly, the structure of the Variable Eccentric Wheels with double eccentric circle is introduced briefly and its maximum height of surmountable obstacle is given at the same time. Then, we analyze the property of the robot from two aspects: walking efficiency and crossing obstacle ability. By using the six ways of action and three states of the robot, the walking efficiency of robot is analyzed, and by adopting the Static Stability Margin Method and Center of Gravity Projection Method, the crossing obstacle ability is analyzed, especially, the ability to climb the stairs in the four eccentric wheels state. And the biggest height limit of slope, which the robot can climb is given. Finally, a series of experiments we did and the analysis of the results prove that the Variable Eccentric Wheel-legged Robot can switch respectively from eccentric to center, and back again, so it can adapt to any terrain keeping high efficiency and making full use of the wheel and eccentric design.
\end{abstract}

\section{Introduction}

The working environment of human beings is unstructured and complex, and the unknown areas are continually explored by humans. At the same time, with the rapid development of China's nuclear power industry, adventure rescue, military reconnaissance, fire fighting vehicle, aerospace, and many other areas, the type of mobile robot, which can walk freely on a flat ground and any other wild environment or complex terrain is needed urgently. There are many types of mobile robot, for example, wheeled robot, tracked robot, multi-legged robot, hybrid robot, and so on [1,2].

But, the tracked robot is bulky and requires high-power drives. The multi-legged robot and hybrid robot are complex and difficult to control. High level of walking efficiency on a flat ground is owned by the wheeled robot, however, at the same time, its obstacle-climbing capability is very poor.

Therefore, in this paper, a model of Variable Eccentric Wheel-legged Robot is presented. The Variable Eccentric Wheel-legged Robot provides a simple and reliable structure, high efficiency, good adaptability to the surface, and more crossing obstacle ability with double eccentric circle, which can maintain both walking stationarity and the efficiency of the power consumption of the system in neat terrain, and in a non-structured topography. The obstacle capability, simple control, high efficiency, low energy consumption and wide application range, are available for the mobile robot depicted above.

\section{The Structure of Variable Eccentric Wheel-legged Robot}

\subsection{Variable Eccentric Wheels with Double Eccentric Circle}

In general, the wheeled mobile robots or the robots with composite structure based on the wheels use a wheel with a round shape, and the center of the wheel in the center position. In Fig. 1(a), when the 
axis of the wheel is in the center position, center wheel is commonly used, and if the axis of the wheel is in the eccentric position, which deviates from circle center, for example, point $O^{\prime}$, a eccentric wheel can be used [3].

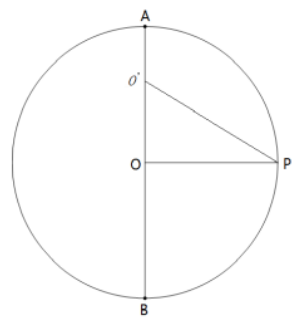

(a)

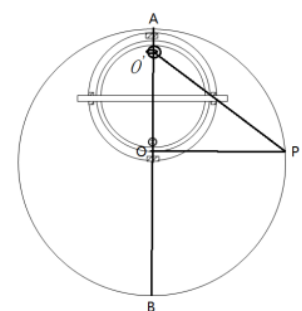

(b)

Figure 1. The difference of eccentric wheel and center wheel.

Therefore, we can combine the characteristics of eccentric wheel and center wheel to put forward a kind of variable eccentric wheel with double eccentric circle (see Fig.1(b)).

The variable eccentric wheel with double eccentric circle is made up of a small circle which can move with dynamic driving and a big circle which can be seen as a driving wheel. In this mechanical structure, power shaft pass point $O^{\prime}$, and there is one limiting device between the small and big circle. With the small wheel rotation, the eccentricity $O O^{\prime}$ will become bigger or smaller.

Through calculation, we can come to a conclusion that the value range of the eccentricity $L$ is $(0$, R). So the maximum height of surmountable obstacle $H$ is

$$
H=R+L-E \text {. }
$$

In this equation, $R$ is the big circle's radius, $E$ is the distance of power shaft and mobile robot's underpan.

In fact, if the robot chassis raises over the big wheel's upper edge, the robot can get stronger ability in crossing obstacle. At this case, the maximum height of surmountable obstacle $H$ is

$$
H=R+L+E \text {. }
$$

\subsection{The Structure of Robot}

The Variable Eccentric Wheel-legged Robot uses a symmetric structure design. It includes four variable eccentric wheels with double eccentric circle and a machine body. There are four same types of electric motors evenly distributed in the machine body; each wheel is driven by one motor independently. Of course, we can design more even number of wheels based on requirement.

There are some extended segments in the front and rear part of the main body of robot. They are used to the main body and double eccentric wheels mutual switch when mobile robot crosses obstacles. The length of the front and rear part must be slightly longer than the radius of wheels to cross obstacles.

Fig. 2 shows a model of the Variable Eccentric Wheel-legged Robot. It can switch patterns simply from eccentric wheels to center wheels, and back again. There are some control units in the body of robot. In short, it owns four featuring compliant wheels and a simple mechanical design.

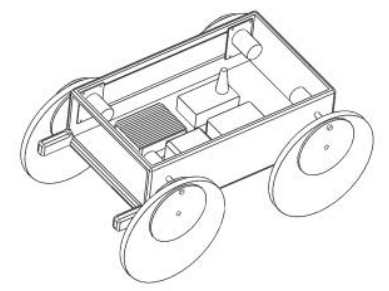

Figure 2. A model of the Variable Eccentric Wheel-legged Robot. 


\section{The Walking Efficiency and Crossing Obstacle Ability of Variable Eccentric Wheel-legged Robot}

In this section we analyze the property of the Variable Eccentric Wheel-legged Robot from two aspects: walking efficiency and crossing obstacle ability [4].

\subsection{Walking Efficiency Analysis}

There are six ways of action of the robot: resetting, moving forwards, moving backwards, turning left, turning right and stopping.

Resetting: This operation will test each functional unit working correctly, and placing any robots at the ground supporting, letting all parts in the ready state waiting for control signals.

Moving forwards and moving backwards: The robot can automatically switch to round wheels mode, and then execute the forward algorithm and back-off algorithm.

Because the four wheels of our robots can switch patterns simply from eccentric wheels to center wheels, and back again, we can get three optimum types of robot's states from experimental results and analysis. They are four round wheels state, four eccentric wheels state, round forward and eccentric back state.

Ordinary round wheel owns the optimal effect of moving forwards and backwards, which have been proved more than three hundred years ago. So, on a flat ground, we can switch to four round wheels state to achieve the greatest walking efficiency. whereas, on a non-flat terrain, we can adopt the round forward and eccentric back state.

Turning left and turning right: The Variable Eccentric Wheel-legged Robot's turning is a complex action and uses a way of differential speed of left and right mechanism. We have to discuss it in several ways.

The four round wheels state owns the greatest turning effect, round forward and eccentric back state take second place, while four eccentric wheels state is the worst one.

Stopping: This operation will call off all driving order of robot until receiving a new signal.

\subsection{Crossing Obstacle Ability Analysis}

When the Variable Eccentric Wheel-legged Robot wants across obstacles, it can be automatically transformed from center wheels state to eccentric wheels state. In this state, it shows excellent capacity of obstacle avoidance.

The crossing obstacle of robots includes three forms: small barriers (eg. square tube, which obstacle's width is smaller than the length of robot's body), platforms (Multiple small obstacles spliced together), and continuous stages (eg. stairs). They are showed respectively in Fig.3 (a), (b), and (c) above. These three types need different condition, parameters and ability that robots own.

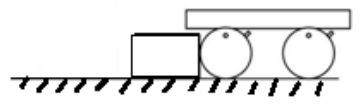

(a)

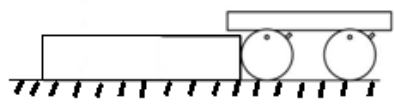

(b)

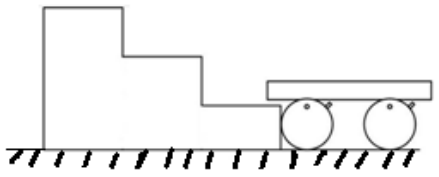

(c)

Figure 3. The forms of the robots' crossing obstacle.

Same as the description of 3.1 section above, there are two eccentric wheels states: four eccentric wheels state, and two eccentric and two center wheels state (eg. round forward and eccentric back state). We come to the conclusion that four eccentric wheels state can get the optimal effect of the robot's continuously crossing of obstacles through the kinematic and dynamic analysis and calculation. But, at the same time, we realize that round forward and eccentric back state can obtain best effect when the robot crosses small barriers. This state is the unique ability of the Variable Eccentric Wheel-legged Robot, which we present in this paper. Because it can maintain the advantages of four eccentric wheels state, and also can obtain the benefits of round forward and eccentric back state. This state is a combination of eccentric wheels and center wheels.

In Fig. 4, we assume that the left and right sides of robot are symmetrical, and the distribution of robot's body weight is uniform. The robot's center of gravity is the center of gravity position in its 
geometric structure. In the following content of this section, we will analyze the obstacle performance of robot through two aspects: static stability and slipping level of slope.

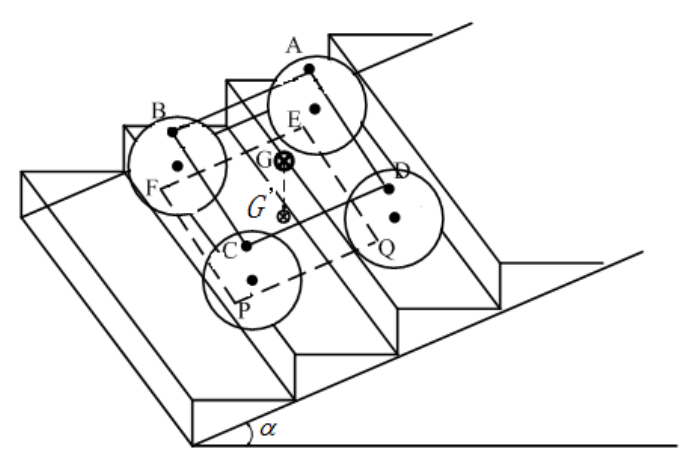

Figure 4. The static stability analysis diagram

The point $G$ ' is the projected point of the robot's center of gravity $G$. Using the Static Stability Margin Method and Center of Gravity Projection Method [5], we can conclude that the robot will keep static stability and cannot turn backwards, when the robot's center of gravity projective position is in the rectangular region EFPQ. The bigger the gradient of stairs or slope $\alpha$, the lower the shift of the projected point of the robot's center of gravity $G^{\prime}$. When the point $G^{\prime}$ is on the line of FP, the climbing slope of the robot is the biggest. For quadruped eccentric wheel-legged robot, the limit height of slope is:

$$
\alpha=\arctan \frac{L}{2(R+2 \mathrm{e})}
$$

In the equation above, $R$ is the big circle's radius, $e$ is the eccentric distance, $L$ is the distance between the front and rear axles.

Assuming that the whole weight of robot is $M$, the friction coefficient between the slope and wheels is $\mu$, we can get the critical value relation of slip phenomenon through mechanical analysis:

$M g \sin \alpha=\mu M g \cos \alpha$

So, the equation (5) is given:

$\alpha=\arctan \mu$

From the above, in the state of four eccentric wheels state, the robot can climb the biggest limit height of slope:

$$
\alpha=\min \left\{\arctan \frac{L}{2(R+2 e)}, \arctan \mu\right\}
$$

From the equation above, the Variable Eccentric Wheel-legged Robot, which is displayed in this paper, can cross all kinds of obstacles which can acquire different gradient $\alpha$ by adjusting the eccentric distance $e$. But, at the same time, we can conclude that the eccentric distance $e$ is not the bigger the better. When the eccentricity $e$ is bigger, the biggest height limit of slope is smaller [6]. So, we should reasonably adjust eccentricity according to the height of the terrain and the obstacles in front and make the barrier effect is best. A conclusion is drawn: this effect and ability are peculiar to the Variable Eccentric Wheel-legged Robot, because only Variable Eccentric Wheel-legged Robot can adjust eccentricity easily at anytime and anywhere.

\section{Experiments and Results}

In this section, in order to verify the walking efficiency and crossing obstacle ability of the Variable Eccentric Wheel-legged Robot, some physical and necessary experiments have been conducted, 
including moving forwards, moving backwards, turning left, turning right, stopping, jumping small barriers, crossing platforms, and climbing continuous stairs, or any other irregular ground.

Crossing obstacles through continuous steps, especially continuous stairs, will be mainly described and analyzed below. The continuous stairs are more difficult for mobile robot.

There are two difficulties to the continuous stairs, compared with a single step [7]. On the one hand, a continuous step has a certain slope gradient, making it necessary for the robots to increase the driving torque. On the other hand, there is a great irregularity comparing with tilted plain, resulting in the structure of the robot should be designed to be simple as much as possible. In this paper, the designed and selected size of stairs is determined according to standard size of the stairs in the building, and other size been also selected which is reasonable and representative [8].

Fig. 5 shows a process of the Variable Eccentric Wheel-legged Robot climbing continuous stairs. Through the experiments, we can conclude that the robot can across stairs easily when it switches to eccentric wheels state. In this pattern, the mutual switching between the body of robot and the eccentric wheels lets the robot complete the climbing process successfully.

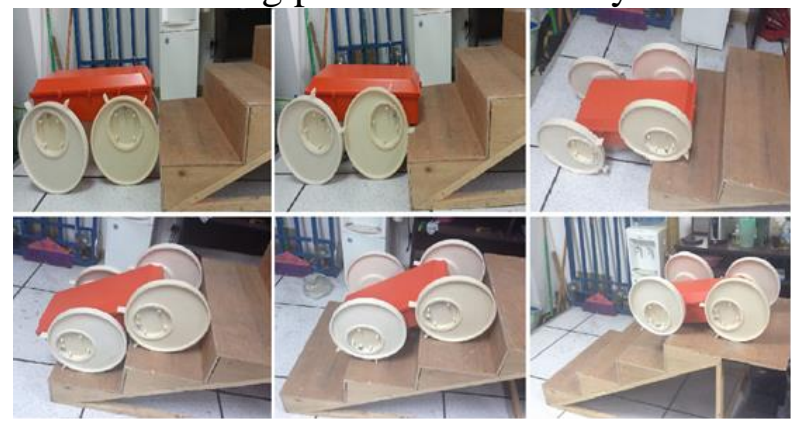

Figure 5. The process of the Variable Eccentric Wheel-legged Robot climbing continuous stairs.

Through a series of experiments to test the stability of robot, we can conclude the experimental and testing results in the following table. For each functional test we repeated $\$ 50 \$$ times.

Table 1. Functional testing experiments of mobile robot.

\begin{tabular}{|c|c|c|c|c|}
\hline States & Functional Testing Experiments & Successes & Failures & Success Rate \\
\hline \multirow{5}{*}{$\begin{array}{l}\text { Four Center } \\
\text { Wheels State }\end{array}$} & Moving Forwards and Backs & 50 & 0 & $100 \%$ \\
\hline & Turning Left and Right & 50 & 0 & $100 \%$ \\
\hline & Stopping & 50 & 0 & $100 \%$ \\
\hline & Crossing Small Barriers & 28 & 22 & $56 \%$ \\
\hline & Climbing Stairs & 0 & 50 & 0 \\
\hline \multirow{5}{*}{$\begin{array}{c}\text { Round Forward } \\
\text { and Eccentric } \\
\text { Back State }\end{array}$} & Moving Forwards and Backs & 50 & 0 & $100 \%$ \\
\hline & Turning Left and Right & 50 & 0 & $100 \%$ \\
\hline & Stopping & 50 & 0 & $100 \%$ \\
\hline & Crossing Small Barriers & 50 & 0 & $100 \%$ \\
\hline & Climbing Stairs & 42 & 8 & $84 \%$ \\
\hline \multirow{5}{*}{$\begin{array}{l}\text { Four Eccentric } \\
\text { Wheels State }\end{array}$} & Moving Forwards and Backs & 50 & 0 & $100 \%$ \\
\hline & Turning Left and Right & 49 & 1 & $98 \%$ \\
\hline & Stopping & 50 & 0 & $100 \%$ \\
\hline & Crossing Small Barriers & 50 & 0 & $100 \%$ \\
\hline & Climbing Stairs & 50 & 0 & $100 \%$ \\
\hline \multirow{2}{*}{ State Switching } & Center to Eccentric Wheel & 50 & 0 & $100 \%$ \\
\hline & Eccentric to Center Wheel & 50 & 0 & $100 \%$ \\
\hline
\end{tabular}

Table 1 shows that the ability of climbing stairs of the four eccentric wheels state is best, the round forward and eccentric back state takes second place, and the four center wheels state is worst, in which state the robot even cannot across obstacle. Though in every state the robot can complete every functional testing except in the four center wheels state climbing stairs, it is obvious that the robot performed rather poorly under some states. For example, under the eccentric wheels state robot cannot complete turning function very well. It warns us that we should switch to corresponding mode to 
complete corresponding function, so that the robot in this paper play the best functional characteristics and perform the best.

\section{Conclusion}

The Variable Eccentric Wheel-legged Robot described in this paper provides a simple and reliable structure, high efficiency, good adaptability to the surface and higher crossing obstacle ability using a variable eccentric wheel with double eccentric circle. The Variable Eccentric Wheel-legged Robot can walk efficiently not only in the neat terrain and keep no ups and downs, but also in a non-neat terrain, under simple control and low energy consumption. Particularly, the powerful obstacle capability remains high in both conditions.

The robot can easily get through three types of obstacles, including small barriers, platforms, and continuous stages, which have been verified through kinematic and dynamic analysis and concrete experiments. When it crosses different types of obstacles, it should adjust to corresponding pattern, through a mechanical device of the variable eccentric wheels with double eccentric circle. In the state of the variable eccentric wheels, the main body of robot and eccentric wheels can switch between each other. On a whole flat ground, it should keep four common round wheels which can keep the stability of walking. But, when it climbs a continuous stairs, it should be switched to four eccentric wheels state, under which the robot can across an obstacle over radius of robot's wheel.

However, it's also worth noting that, when the robot crosses a ground which is flat for a while, and smooth for a while, that is uneven, small ups and downs of the ground, it is best to switch to the round forward and eccentric back state. In this state, robots can both keep walking not ups and downs, and efficiently over the obstacles on the ground, and, at the same time, it doesn't need to often switch between the eccentric wheels and center wheels. In addition, at this ground and in the state depicted above, the robot can turn left and right easily.

The four wheels of the Variable Eccentric Wheel-legged Robot can switch respectively from eccentric to center, and back again, so it can adapt to any terrain keeping high efficiency and making full use of the wheel and eccentric. In the next step of work, the material and structure of parts will be optimized and more testing experiments will need to be done.

\section{Acknowledgements}

This work is financially supported by "Innovative Research Project for Postgraduate Students of Chongqing, China"(CYS2015050) and "Fundamental Research Funds for the Central Universities" (XDJK2016D022, XDJK2017D063, XDJK2017D070).

\section{References}

[1] LI Lei, YE Tao, TAN Min, CHEN Xi jun. Present State and Future Development of Mobile Robot Technology Research[J]. Robot, Vol. 24, No. 5, p. 475-480.

[2] ZHANG Ming-lu, DING Cheng-jun, DUAN Ping. Present Situation and Future Development of Mobile Robot Research [J]. Journal of Hebei University of Technology. Vol. 33, No. 2, p. 100-114.

[3] GE Geng-yu, WANG Yu-jun. On Obstacle Crossing Research for Quadruped Eccentric Wheeled-Legged Robot [J]. Journal of Southwest China Normal University (Natural Science Edition). Vol. 39, No. 10, p. 95-100.

[4] Hu Xiang: Small Robot Climbing the Ladder of Continuous [D]. Master Degree, Southwest University, China, 2015. p. 24-29.

[5] LI Jun-ke, WANG Yu-jun, et al. Static Stability Analysis on Three-Foot Gait of Eccentric-Type Legged Hexapod Robot [J]. Journal of Henan Institute of Education (Natural Science Edition). Vol. 21, No. 1, p. 40-43. 
[6] E. z. Moore, D. Campbell, et al. Reliable Stairs Climbing in the Simple Hexapod 'Rhex'[C]. IEEE Int. Conf. Robotics and Automation (ICRA), April, 2004. P. 2153-2158.

[7] Bruno Siciliano, Oussama Khatib. Handbook of Robotics [M]. Peking: China Machine Press. 2013:641-642.

[8] YU Yong-chuan, YUAN Kui, ZOU Wei. Dynamic Model of All-Wheel-Drive Mobile Robot Climbing over Obstacles and Analysis on Its Influential Factors [J]. Robot, Vol. 30, No. 1, p. 1-6. 